INTERNATIONAL JOURNAL OF MULTIDISCIPLINARY RESEARCH AND ANALYSIS

ISSN(print): 2643-9840, ISSN(online): 2643-9875

Volume 04 Issue 12 December 2021

DOI: 10.47191/ijmra/v4-i12-20, Impact Factor: 6.072

Page No.- 1906-1916

\title{
The Relative Global Consequences of Cumulative Distribution of Covid-19, Using the USA as Comparism Factor and Cumulative Covid -19 Data of 31 ${ }^{\text {st }}$ October 2021
}

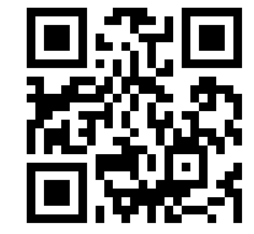

\author{
Joseph Oyepata Simeon ${ }^{1}$, Joseph Opeyemi Tosin ${ }^{2}$, Adeoye David Adegbenga ${ }^{3}$ \\ ${ }^{1.3}$ Departmennt of Pharmacology and Toxicology, Faculty of Pharmaceutical Sciences, Federal University, Oye-Ekiti, Ekiti State, \\ Nigeria \\ ${ }^{2}$ Department of Pharmacy, University College Hospital, Ibadan, Oyo State, Nigeria
}

\begin{abstract}
COVID -19 have affected over 200 million and killed about 5 million people globally. Many steps have been taken to understand its dynamics, etiology and infectivity. Several approach have also been taken to control and manage the virus, while vaccines have been developed to prevent the rate of infectivity. Because of complete lack of knowledge of the virus, shortage in vaccine supply etc, understanding how the virus spread per country may determine relativity in vaccine emergency. The aim of this study is to determine the relative global consequences of cumulative distribution of Covid-19, using the USA as comparism factor and cumulative covid -19 data of 31st October 2021. Data from one hundred and fifty four countries were selected based on their continents, countries and cases of infection. Data were obtained from United Nations Geoscheme and WHO. They were analyzed and compared to that of the United State of America (USA) value. Data analyzed showed that most Africa countries are least to be affected while Americans and Europeans appear to be most affected. Result from the study shows that Africa may have develop mechanisms to cope and survive the virus pandemic compared to other region of the world. Hence, vaccine priority may be considered base on infectivity and severity of infection.
\end{abstract}

KEYWORD: Africa, USA, COVID-19, countries, continent

\section{INTRODUCTION}

Coronavirus disease 2019 (COVID-19) is a contagious disease caused by severe acute respiratory syndrome coronavirus 2 (SARSCoV-2) [1]. The first known case was identified in Wuhan, China, in December 2019[2]. The disease has since spread worldwide, leading to an ongoing pandemic. Symptoms of COVID-19 are variable, but often include cough, fever [3], difficulties in breathing, headache [4], fatigue, and loss of smell and taste [5]. Symptoms usually begin one to fourteen days after been exposured to the virus. At least a third of people who are infected do not develop noticeable symptoms [6]. Of those people who develop symptoms noticeable enough to be classed as patients, most ( $81 \%$ ) develop mild to moderate symptoms (up to mild pneumonia), while $14 \%$ develop severe symptoms (dyspnea, hypoxia, or more than $50 \%$ lung involvement on imaging), and $5 \%$ suffer critical symptoms (respiratory failure, shock, or multiorgan dysfunction) [7]. Older people are usually at a higher risk of developing severe symptoms. Some people, continue to experience a range of effects (long COVID) for months after recovery, and damage to organs has been observed [8]. Multi-year studies are underway to further investigate the long-term effects of the disease [9].

A COVID-19 vaccine is a vaccine intended to provide acquired immunity against severe acute respiratory syndrome coronavirus 2 (SARS-CoV-2), the virus that causes coronavirus disease 2019 (COVID-19). Prior to the COVID-19 pandemic, an little knowledge existed about the structure and function of coronaviruses causing diseases like severe acute respiratory syndrome (SARS) and Middle East respiratory syndrome (MERS) $[10,11]$. This knowledge accelerated the development of various vaccine platforms in early 2020[12]. The initial focus of SARS-CoV-2 vaccines was on preventing symptomatic, often severe illness [13]. On 10 January 2020, the SARS-CoV-2 genetic sequence data was shared through GISAID, and by 19 March, the global pharmaceutical industry announced a major commitment to address COVID-19 [14]. The COVID-19 vaccines are widely credited for their role in reducing the spread, severity, and death caused by COVID-19. Many countries have implemented phased distribution plans that prioritize those at highest risk of complications, such as the elderly, and those at high risk of exposure and transmission, such as 


\section{The Relative Global Consequences of Cumulative Distribution of Covid-19, Using the USA as Comparism Factor and Cumulative Covid -19 Data of 31 ${ }^{\text {st }}$ October 2021}

healthcare workers [15]. Recently, recommendations from several institution and research groups have been approved for the vaccination to be extended to children[16].

Multiple variants of the virus that causes COVID-19 have been documented globally, during this pandemic. In the United Kingdom (UK), a new variant has emerged with an unusually large number of mutations. In South Africa, another variant has emerged independently of the variant detected in the UK. Another variant recently emerged in Nigeria and USA[17]. Delta variant was found to be more infectious and was leading to increased transmissibility when compared with other variants, even in some vaccinated individuals[18]. The different waves of the disease has been of concern due to change in weather and mutated strain of the virus identified in some countries[18,19]. There is the need to understand this surge with the virulent and spreading ability of the newly mutated strain of the virus. Also, Several study has been carried out on the dermographic strength and nature of the virus, but analyzing an updated information per time is very essential in managing the trend. The aim of this study is to determine the relative global consequences of cumulative distribution of Covid-19, using the USA as comparism factor and cumulative covid -19 data of $31^{\text {st }}$ October 2021.

\section{MATERIAL AND METHOD}

A total of one hundred and fifty four (154) countries from different continent and regions of the world were selected from different continents, based on COVID-19 cases in each country. The listed countries and territories with their continental regional classification were based on the United Nations Geoscheme and WHO. Sources and data used were provided under Latest Updates from WHO/World meter's on October $31,2021^{8}$. Data obtained for each country cumulative total per 100000 populations was analyzed and directly compared to that of the United State of America (USA). USA was used as a Comparism Factor (CF) because it has one of the best healthcare systems and still highest COVID-19 cases in the world. All data used in these analyses are from publicly available data sets.

\section{STATISTICAL ANALYSIS}

Parameters such as cumulative incidences/cases and cumulative deaths of countries per 100000 population were compared against factors obtained for USA. Bivariate analysis, was done with Chi-square test to compare proportions for variables. In reporting these results, country-level characteristics are scaled to represent a comparison of two countries similar in all other respects. Thus, rate ratios greater than one means that higher levels of a given characteristic are associated with higher rates of COVID-19 cases or deaths, while rate ratios less than one means that lower levels of a given characteristic are associated with lower rates of COVID-19 cases or deaths.

\section{RESULT}

based on analysed data, with exception of south Africa, Africa is unbotherly affected by the virus when compared to other continents with regards to incidence and death cases when compared to values obtained for the USA. America and Europe have the most infection to population ratio. Result also showed most countries of the world have considerable number of affected citizens (Table 1).

Table 1: Infectious, recovery and mortality rate of COVID-19 based on country

\begin{tabular}{|c|c|c|c|c|c|c|c|c|}
\hline & Name & WHO Region & Cases - CT & $\begin{array}{l}\text { Cases - } \\
\text { CTPMP (A) }\end{array}$ & $\begin{array}{l}\text { Deaths - } \\
\text { CT }\end{array}$ & $\begin{array}{l}\text { Deaths - } \\
\text { CTPMP } \\
\text { (B) }\end{array}$ & $\begin{array}{l}A / 13787.1 \\
(C F 1)\end{array}$ & $\begin{array}{l}B / 223.5 \\
(C F 2)\end{array}$ \\
\hline 1 & USA & Americas & 45635708 & 13787.11 & 739856.00 & 223.52 & 1.00 & 1.00 \\
\hline 2 & India & South-East Asia & 34285814 & 2484.47 & 458437.00 & 33.22 & 0.18 & 0.15 \\
\hline 3 & Brazil & Americas & 21804094 & 10257.88 & 607694.00 & 285.89 & 0.74 & 1.28 \\
\hline 4 & The United Kingdom & Europe & 9019966 & 13286.93 & 140558.00 & 207.05 & 0.96 & 0.93 \\
\hline 5 & Russian Federation & Europe & 8513790 & 5833.98 & 238538.00 & 163.46 & 0.42 & 0.73 \\
\hline 6 & Turkey & Europe & 8009040 & 9496.24 & 70410.00 & 83.48 & 0.69 & 0.37 \\
\hline 7 & France & Europe & 6937450 & 10666.54 & 115265.00 & 177.22 & 0.77 & 0.79 \\
\hline 8 & Iran & $\begin{array}{l}\text { Eastern } \\
\text { Mediterranean }\end{array}$ & 5916211 & 7043.70 & 126126.00 & 150.16 & 0.51 & 0.67 \\
\hline 9 & Argentina & Americas & 5288807 & 11702.00 & 115950.00 & 256.55 & 0.85 & 1.15 \\
\hline
\end{tabular}


The Relative Global Consequences of Cumulative Distribution of Covid-19, Using the USA as Comparism Factor and Cumulative Covid -19 Data of $31^{\text {st }}$ October 2021

\begin{tabular}{|c|c|c|c|c|c|c|c|c|}
\hline 10 & Spain & Europe & 5011149 & 10587.10 & 87368.00 & 184.58 & 0.77 & 0.83 \\
\hline 11 & Colombia & Americas & 5000677 & 9827.82 & 127258.00 & 250.10 & 0.71 & 1.12 \\
\hline 12 & Italy & Europe & 4767440 & 7993.50 & 132074.00 & 221.45 & 0.58 & 0.99 \\
\hline 13 & Germany & Europe & 4597550 & 5528.11 & 95729.00 & 115.11 & 0.40 & 0.52 \\
\hline 14 & Indonesia & South-East Asia & 4244358 & 1551.73 & 143405.00 & 52.43 & 0.11 & 0.23 \\
\hline 15 & Mexico & Americas & 3805765 & 2951.74 & 288276.00 & 223.59 & 0.21 & 1.00 \\
\hline 16 & Poland & Europe & 3025247 & 7969.96 & 76999.00 & 202.85 & 0.58 & 0.91 \\
\hline 17 & Ukraine & Europe & 2922302 & 6682.03 & 67729.00 & 154.87 & 0.48 & 0.69 \\
\hline 18 & South Africa & Africa & 2921886 & 4926.57 & 89163.00 & 150.34 & 0.36 & 0.67 \\
\hline 19 & Philippines & Western Pacific & 2787276 & 2543.57 & 43172.00 & 39.40 & 0.18 & 0.18 \\
\hline 20 & Malaysia & Western Pacific & 2471642 & 7636.54 & 28912.00 & 89.33 & 0.55 & 0.40 \\
\hline 21 & Peru & Americas & 2200908 & 6675.11 & 200217.00 & 607.24 & 0.48 & 2.72 \\
\hline 22 & Netherlands & Europe & 2123880 & 12200.89 & 18397.00 & 105.68 & 0.88 & 0.47 \\
\hline 23 & Iraq & $\begin{array}{l}\text { Eastern } \\
\text { Mediterranean }\end{array}$ & 2054202 & 5107.10 & 23138.00 & 57.53 & 0.37 & 0.26 \\
\hline 24 & Thailand & South-East Asia & 1920189 & 2750.99 & 19260.00 & 27.59 & 0.20 & 0.12 \\
\hline 25 & Czechia & Europe & 1762902 & 16485.06 & 30753.00 & 287.57 & 1.20 & 1.29 \\
\hline 26 & Japan & Western Pacific & 1722864 & 1362.20 & 18268.00 & 14.44 & 0.10 & 0.06 \\
\hline 27 & Canada & Americas & 1712128 & 4536.38 & 28952.00 & 76.71 & 0.33 & 0.34 \\
\hline 28 & Chile & Americas & 1695048 & 8867.08 & 37757.00 & 197.51 & 0.64 & 0.88 \\
\hline 29 & Romania & Europe & 1648031 & 8526.28 & 47751.00 & 247.05 & 0.62 & 1.11 \\
\hline 30 & Bangladesh & South-East Asia & 1569539 & 953.03 & 27868.00 & 16.92 & 0.07 & 0.08 \\
\hline 31 & Belgium & Europe & 1360650 & 11808.70 & 25994.00 & 225.60 & 0.86 & 1.01 \\
\hline 32 & Israel & Europe & 1326966 & 15330.83 & 8085.00 & 93.41 & 1.11 & 0.42 \\
\hline 33 & Pakistan & $\begin{array}{l}\text { Eastern } \\
\text { Mediterranean }\end{array}$ & 1272345 & 576.00 & 28439.00 & 12.88 & 0.04 & 0.06 \\
\hline 34 & Sweden & Europe & 1171512 & 11343.52 & 15025.00 & 145.48 & 0.82 & 0.65 \\
\hline 35 & Serbia & Europe & 1137820 & 16426.57 & 9890.00 & 142.78 & 1.19 & 0.64 \\
\hline 36 & Portugal & Europe & 1089888 & 10585.64 & 18156.00 & 176.34 & 0.77 & 0.79 \\
\hline 37 & Kazakhstan & Europe & 1018841 & 5426.09 & 17131.00 & 91.24 & 0.39 & 0.41 \\
\hline 38 & Cuba & Americas & 952001 & 8404.99 & 8236.00 & 72.71 & 0.61 & 0.33 \\
\hline 39 & Morocco & $\begin{array}{l}\text { Eastern } \\
\text { Mediterranean }\end{array}$ & 945960 & 2562.84 & 14667.00 & 39.74 & 0.19 & 0.18 \\
\hline 40 & Viet Nam & Western Pacific & 921122 & 946.31 & 22083.00 & 22.69 & 0.07 & 0.10 \\
\hline 41 & Switzerland & Europe & 868896 & 10039.68 & 10802.00 & 124.81 & 0.73 & 0.56 \\
\hline 42 & Hungary & Europe & 863419 & 8837.88 & 30729.00 & 314.54 & 0.64 & 1.41 \\
\hline 43 & Jordan & $\begin{array}{l}\text { Eastern } \\
\text { Mediterranean }\end{array}$ & 860818 & 8436.80 & 11028.00 & 108.08 & 0.61 & 0.48 \\
\hline 44 & Austria & Europe & 822264 & 9237.82 & 11072.00 & 124.39 & 0.67 & 0.56 \\
\hline 45 & Nepal & South-East Asia & 812570 & 2788.81 & 11407.00 & 39.15 & 0.20 & 0.18 \\
\hline 46 & United Arab Emirates & $\begin{array}{l}\text { Eastern } \\
\text { Mediterranean }\end{array}$ & 739824 & 7480.22 & 2136.00 & 21.60 & 0.54 & 0.10 \\
\hline 47 & Greece & Europe & 739448 & 6898.76 & 15894.00 & 148.29 & 0.50 & 0.66 \\
\hline 48 & Georgia & Europe & 719247 & 18029.97 & 10045.00 & 251.81 & 1.31 & 1.13 \\
\hline 49 & Tunisia & $\begin{array}{l}\text { Eastern } \\
\text { Mediterranean }\end{array}$ & 712581 & 6029.31 & 25238.00 & 213.54 & 0.44 & 0.96 \\
\hline
\end{tabular}


The Relative Global Consequences of Cumulative Distribution of Covid-19, Using the USA as Comparism Factor and Cumulative Covid -19 Data of 31 ${ }^{\text {st }}$ October 2021

\begin{tabular}{|c|c|c|c|c|c|c|c|c|}
\hline 50 & Lebanon & $\begin{array}{l}\text { Eastern } \\
\text { Mediterranean }\end{array}$ & 641339 & 9396.30 & 8495.00 & 124.46 & 0.68 & 0.56 \\
\hline 51 & Guatemala & Americas & 601402 & 3356.87 & 15050.00 & 84.01 & 0.24 & 0.38 \\
\hline 52 & Bulgaria & Europe & 601035 & 8646.14 & 23918.00 & 344.07 & 0.63 & 1.54 \\
\hline 53 & Belarus & Europe & 598132 & 6329.89 & 4614.00 & 48.83 & 0.46 & 0.22 \\
\hline 54 & Costa Rica & Americas & 559698 & 10987.14 & 7029.00 & 137.98 & 0.80 & 0.62 \\
\hline 55 & Saudi Arabia & $\begin{array}{l}\text { Eastern } \\
\text { Mediterranean }\end{array}$ & 548571 & 1575.73 & 8793.00 & 25.26 & 0.11 & 0.11 \\
\hline 56 & Sri Lanka & South-East Asia & 541073 & 2526.81 & 13743.00 & 64.18 & 0.18 & 0.29 \\
\hline 57 & Azerbaijan & Europe & 529109 & 5218.46 & 7049.00 & 69.52 & 0.38 & 0.31 \\
\hline 58 & Ecuador & Americas & 515859 & 2923.87 & 32958.00 & 186.80 & 0.21 & 0.84 \\
\hline 59 & $\begin{array}{l}\text { Bolivia (Plurinational } \\
\text { State of) }\end{array}$ & Americas & 513584 & 4399.75 & 18925.00 & 162.13 & 0.32 & 0.73 \\
\hline 60 & Myanmar & South-East Asia & 500073 & 919.09 & 18697.00 & 34.36 & 0.07 & 0.15 \\
\hline 61 & Slovakia & Europe & 483773 & 8863.76 & 13034.00 & 238.81 & 0.64 & 1.07 \\
\hline 62 & Panama & Americas & 472534 & 10951.55 & 7315.00 & 169.53 & 0.79 & 0.76 \\
\hline 63 & Croatia & Europe & 470348 & 11590.17 & 9220.00 & 227.20 & 0.84 & 1.02 \\
\hline 64 & Paraguay & Americas & 461006 & 6463.42 & 16246.00 & 227.77 & 0.47 & 1.02 \\
\hline 65 & Ireland & Europe & 443631 & 8936.17 & 5436.00 & 109.50 & 0.65 & 0.49 \\
\hline 66 & Kuwait & $\begin{array}{l}\text { Eastern } \\
\text { Mediterranean }\end{array}$ & 412653 & 9662.71 & 2461.00 & 57.63 & 0.70 & 0.26 \\
\hline 67 & Lithuania & Europe & 408715 & 14627.84 & 5873.00 & 210.19 & 1.06 & 0.94 \\
\hline 68 & $\begin{array}{l}\text { Venezuela (Bolivarian } \\
\text { Republic of) }\end{array}$ & Americas & 406239 & 1428.61 & 4884.00 & 17.18 & 0.10 & 0.08 \\
\hline 69 & Uruguay & Americas & 393718 & 11334.16 & 6077.00 & 174.94 & 0.82 & 0.78 \\
\hline 70 & Denmark & Europe & 386251 & 6633.47 & 2713.00 & 46.59 & 0.48 & 0.21 \\
\hline 71 & Honduras & Americas & 375381 & 3789.96 & 10240.00 & 103.39 & 0.27 & 0.46 \\
\hline 72 & Republic of Korea & Western Pacific & 366386 & 714.63 & 2859.00 & 5.58 & 0.05 & 0.02 \\
\hline 73 & Ethiopia & Africa & 364960 & 317.46 & 6451.00 & 5.61 & 0.02 & 0.03 \\
\hline 74 & Libya & $\begin{array}{l}\text { Eastern } \\
\text { Mediterranean }\end{array}$ & 356655 & 5190.51 & 5078.00 & 73.90 & 0.38 & 0.33 \\
\hline 75 & Mongolia & Western Pacific & 346490 & 10569.23 & 1638.00 & 49.97 & 0.77 & 0.22 \\
\hline 76 & Republic of Moldova & Europe & 337115 & 8356.92 & 7751.00 & 192.14 & 0.61 & 0.86 \\
\hline 77 & Slovenia & Europe & 334188 & 15945.14 & 5079.00 & 242.34 & 1.16 & 1.08 \\
\hline 78 & Egypt & $\begin{array}{l}\text { Eastern } \\
\text { Mediterranean }\end{array}$ & 330084 & 322.55 & 18592.00 & 18.17 & 0.02 & 0.08 \\
\hline 79 & Armenia & Europe & 306739 & 10351.49 & 6284.00 & 212.07 & 0.75 & 0.95 \\
\hline 80 & Oman & $\begin{array}{l}\text { Eastern } \\
\text { Mediterranean }\end{array}$ & 304281 & 5958.55 & 4111.00 & 80.50 & 0.43 & 0.36 \\
\hline 81 & Bahrain & $\begin{array}{l}\text { Eastern } \\
\text { Mediterranean }\end{array}$ & 276794 & 16266.93 & 1393.00 & 81.87 & 1.18 & 0.37 \\
\hline 82 & $\begin{array}{ll}\text { Bosnia } & \text { and } \\
\text { Herzegovina } & \end{array}$ & Europe & 253757 & 7734.57 & 11528.00 & 351.38 & 0.56 & 1.57 \\
\hline 83 & Kenya & Africa & 253293 & 471.06 & 5276.00 & 9.81 & 0.03 & 0.04 \\
\hline 84 & Qatar & $\begin{array}{l}\text { Eastern } \\
\text { Mediterranean }\end{array}$ & 239143 & 8300.54 & 610.00 & 21.17 & 0.60 & 0.09 \\
\hline 85 & Nigeria & Africa & 211887 & 102.79 & 2895.00 & 1.40 & 0.01 & 0.01 \\
\hline
\end{tabular}


The Relative Global Consequences of Cumulative Distribution of Covid-19, Using the USA as Comparism Factor and Cumulative Covid -19 Data of $31^{\text {st }}$ October 2021

\begin{tabular}{|c|c|c|c|c|c|c|c|c|}
\hline 86 & Zambia & Africa & 209722 & 1140.79 & 3661.00 & 19.91 & 0.08 & 0.09 \\
\hline 87 & Algeria & Africa & 206358 & 470.59 & 5918.00 & 13.50 & 0.03 & 0.06 \\
\hline 88 & Norway & Europe & 203742 & 3795.79 & 900.00 & 16.77 & 0.28 & 0.08 \\
\hline 89 & North Macedonia & Europe & 202203 & 9705.53 & 7121.00 & 341.80 & 0.70 & 1.53 \\
\hline 90 & Singapore & Western Pacific & 198374 & 3390.81 & 407.00 & 6.96 & 0.25 & 0.03 \\
\hline 91 & Botswana & Africa & 186594 & 7934.68 & 2406.00 & 102.31 & 0.58 & 0.46 \\
\hline 92 & Uzbekistan & Europe & 185963 & 555.62 & 1323.00 & 3.95 & 0.04 & 0.02 \\
\hline 93 & Albania & Europe & 184887 & 6424.60 & 2916.00 & 101.33 & 0.47 & 0.45 \\
\hline 94 & Puerto Rico & Americas & 184812 & 6460.03 & 3234.00 & 113.04 & 0.47 & 0.51 \\
\hline 95 & Kyrgyzstan & Europe & 181259 & 2778.26 & 2671.00 & 40.94 & 0.20 & 0.18 \\
\hline 96 & Australia & Western Pacific & 170458 & 668.47 & 1734.00 & 6.80 & 0.05 & 0.03 \\
\hline 97 & Finland & Europe & 157531 & 2851.09 & 1158.00 & 20.96 & 0.21 & 0.09 \\
\hline 98 & Afghanistan & $\begin{array}{l}\text { Eastern } \\
\text { Mediterranean }\end{array}$ & 156210 & 401.28 & 7272.00 & 18.68 & 0.03 & 0.08 \\
\hline 99 & Mozambique & Africa & 151288 & 484.04 & 1930.00 & 6.18 & 0.04 & 0.03 \\
\hline 100 & Montenegro & Europe & 143762 & 22889.78 & 2095.00 & 333.57 & 1.66 & 1.49 \\
\hline 101 & Zimbabwe & Africa & 132954 & 894.54 & 4677.00 & 31.47 & 0.06 & 0.14 \\
\hline 102 & Ghana & Africa & 130077 & 418.62 & 1175.00 & 3.78 & 0.03 & 0.02 \\
\hline 103 & Namibia & Africa & 128886 & 5072.45 & 3552.00 & 139.79 & 0.37 & 0.63 \\
\hline 104 & Uganda & Africa & 126171 & 275.84 & 3215.00 & 7.03 & 0.02 & 0.03 \\
\hline 105 & China & Western Pacific & 126078 & 8.57 & 5696.00 & 0.39 & 0.00 & 0.00 \\
\hline 106 & Cyprus & Europe & 124332 & 14001.27 & 574.00 & 64.64 & 1.02 & 0.29 \\
\hline 107 & Cambodia & Western Pacific & 118522 & 708.91 & 2788.00 & 16.68 & 0.05 & 0.07 \\
\hline 108 & Cameroon & Africa & 102499 & 386.12 & 1686.00 & 6.35 & 0.03 & 0.03 \\
\hline 109 & Rwanda & Africa & 99681 & 769.61 & 1328.00 & 10.25 & 0.06 & 0.05 \\
\hline 110 & Jamaica & Americas & 89014 & 3006.05 & 2236.00 & 75.51 & 0.22 & 0.34 \\
\hline 111 & Maldives & South-East Asia & 87784 & 16239.94 & 243.00 & 44.96 & 1.18 & 0.20 \\
\hline 112 & Senegal & Africa & 73917 & 441.46 & 1878.00 & 11.22 & 0.03 & 0.05 \\
\hline 113 & Angola & Africa & 64374 & 195.87 & 1708.00 & 5.20 & 0.01 & 0.02 \\
\hline 114 & Malawi & Africa & 61794 & 323.02 & 2300.00 & 12.02 & 0.02 & 0.05 \\
\hline 115 & CÃ'te dâ $€^{\mathrm{TM}} \mid$ voire & Africa & 61292 & 232.36 & 695.00 & 2.64 & 0.02 & 0.01 \\
\hline 116 & $\begin{array}{l}\text { Democratic Republic } \\
\text { of the Congo }\end{array}$ & Africa & 57556 & 64.26 & 1098.00 & 1.23 & 0.00 & 0.01 \\
\hline 117 & Trinidad and Tobago & Americas & 57329 & 4096.43 & 1696.00 & 121.19 & 0.30 & 0.54 \\
\hline 118 & Madagascar & Africa & 43626 & 157.55 & 963.00 & 3.48 & 0.01 & 0.02 \\
\hline 119 & Syrian Arab Republic & $\begin{array}{l}\text { Eastern } \\
\text { Mediterranean }\end{array}$ & 43146 & 246.54 & 2560.00 & 14.63 & 0.02 & 0.07 \\
\hline 120 & Sudan & $\begin{array}{l}\text { Eastern } \\
\text { Mediterranean }\end{array}$ & 40433 & 92.21 & 2995.00 & 6.83 & 0.01 & 0.03 \\
\hline 121 & Malta & Europe & 37639 & 7314.74 & 461.00 & 89.59 & 0.53 & 0.40 \\
\hline 122 & Mauritania & Africa & 37222 & 800.53 & 794.00 & 17.08 & 0.06 & 0.08 \\
\hline 123 & Gabon & Africa & 35525 & 1596.10 & 239.00 & 10.74 & 0.12 & 0.05 \\
\hline 124 & Guinea & Africa & 30653 & 233.41 & 385.00 & 2.93 & 0.02 & 0.01 \\
\hline 125 & Papua New Guinea & Western Pacific & 29715 & 332.12 & 370.00 & 4.14 & 0.02 & 0.02 \\
\hline 126 & $\begin{array}{l}\text { United Republic of } \\
\text { Tanzania }\end{array}$ & Africa & 26154 & 43.78 & 725.00 & 1.21 & 0.00 & 0.01 \\
\hline
\end{tabular}


The Relative Global Consequences of Cumulative Distribution of Covid-19, Using the USA as Comparism Factor and Cumulative Covid -19 Data of 31 ${ }^{\text {st }}$ October 2021

\begin{tabular}{|c|c|c|c|c|c|c|c|c|}
\hline 127 & Togo & Africa & 26068 & 314.88 & 242.00 & 2.92 & 0.02 & 0.01 \\
\hline 128 & Benin & Africa & 24749 & 204.15 & 161.00 & 1.33 & 0.01 & 0.01 \\
\hline 129 & Haiti & Americas & 23823 & 208.93 & 662.00 & 5.81 & 0.02 & 0.03 \\
\hline 130 & Bahamas & Americas & 22396 & 5695.19 & 643.00 & 163.51 & 0.41 & 0.73 \\
\hline 131 & Somalia & $\begin{array}{l}\text { Eastern } \\
\text { Mediterranean }\end{array}$ & 21998 & 138.41 & 1208.00 & 7.60 & 0.01 & 0.03 \\
\hline 132 & Lesotho & Africa & 21635 & 1009.92 & 658.00 & 30.72 & 0.07 & 0.14 \\
\hline 133 & Burundi & Africa & 20078 & 168.85 & 14.00 & 0.12 & 0.01 & 0.00 \\
\hline 134 & Mauritius & Africa & 17812 & 1400.57 & 176.00 & 13.84 & 0.10 & 0.06 \\
\hline 135 & Barbados & Americas & 17763 & 6181.12 & 153.00 & 53.24 & 0.45 & 0.24 \\
\hline 136 & Congo & Africa & 17670 & 320.22 & 278.00 & 5.04 & 0.02 & 0.02 \\
\hline 137 & Mali & Africa & 16039 & 79.20 & 563.00 & 2.78 & 0.01 & 0.01 \\
\hline 138 & Burkina Faso & Africa & 14793 & 70.77 & 214.00 & 1.02 & 0.01 & 0.00 \\
\hline 139 & Iceland & Europe & 13492 & 3705.23 & 33.00 & 9.06 & 0.27 & 0.04 \\
\hline 140 & Djibouti & $\begin{array}{l}\text { Eastern } \\
\text { Mediterranean }\end{array}$ & 13478 & 1364.17 & 181.00 & 18.32 & 0.10 & 0.08 \\
\hline 141 & Equatorial Guinea & Africa & 13368 & 952.83 & 167.00 & 11.90 & 0.07 & 0.05 \\
\hline 142 & Nicaragua & Americas & 12866 & 194.22 & 208.00 & 3.14 & 0.01 & 0.01 \\
\hline 143 & South Sudan & Africa & 12402 & 110.79 & 133.00 & 1.19 & 0.01 & 0.01 \\
\hline 144 & $\begin{array}{ll}\text { Central } & \text { African } \\
\text { Republic } & \end{array}$ & Africa & 11579 & 239.74 & 100.00 & 2.07 & 0.02 & 0.01 \\
\hline 145 & Gambia & Africa & 9967 & 412.43 & 340.00 & 14.07 & 0.03 & 0.06 \\
\hline 146 & Yemen & $\begin{array}{l}\text { Eastern } \\
\text { Mediterranean }\end{array}$ & 9779 & 32.79 & 1880.00 & 6.30 & 0.00 & 0.03 \\
\hline 147 & Eritrea & Africa & 6816 & 192.19 & 45.00 & 1.27 & 0.01 & 0.01 \\
\hline 148 & Sierra Leone & Africa & 6398 & 80.21 & 121.00 & 1.52 & 0.01 & 0.01 \\
\hline 149 & Niger & Africa & 6339 & 26.19 & 212.00 & 0.88 & 0.00 & 0.00 \\
\hline 150 & New Zealand & Western Pacific & 6233 & 129.26 & 28.00 & 0.58 & 0.01 & 0.00 \\
\hline 151 & Guinea-Bissau & Africa & 6133 & 311.64 & 141.00 & 7.17 & 0.02 & 0.03 \\
\hline 152 & Liberia & Africa & 5812 & 114.91 & 287.00 & 5.68 & 0.01 & 0.03 \\
\hline 153 & Bermuda & Americas & 5647 & 9068.14 & 101.00 & 162.19 & 0.66 & 0.73 \\
\hline 154 & Chad & Africa & 5069 & 30.86 & 174.00 & 1.06 & 0.00 & 0.00 \\
\hline
\end{tabular}

Key: $\quad$ CT= Cummulative Total

CTPMP= cumulative total per 100000 population

Sources and data used were provided under Latest Updates from WHO/World meter's on 31 $1^{\text {st }}$ October, 2021

Figures obtained for USA were used as the comparism factor (CF), which is a ratio of figure obtained to the respective country population divided by the value obtained for USA.

Values of CF1 and CF2 represent case/incidence and mortality index.

Factor of more than 1 = very high infection and mortality index

Factor of approximately $1=$ high infection and mortality index

Factor of $\leq 1$ but $\geq 0.5=$ moderately high infection and mortality index

Factor of $\leq 0.5$ but $\geq 0.1$ = low infection and mortality index

Factor of $<0.1$ = very low infection, mortality and recovery index 
The Relative Global Consequences of Cumulative Distribution of Covid-19, Using the USA as Comparism Factor and Cumulative Covid -19 Data of 31 ${ }^{\text {st }}$ October 2021

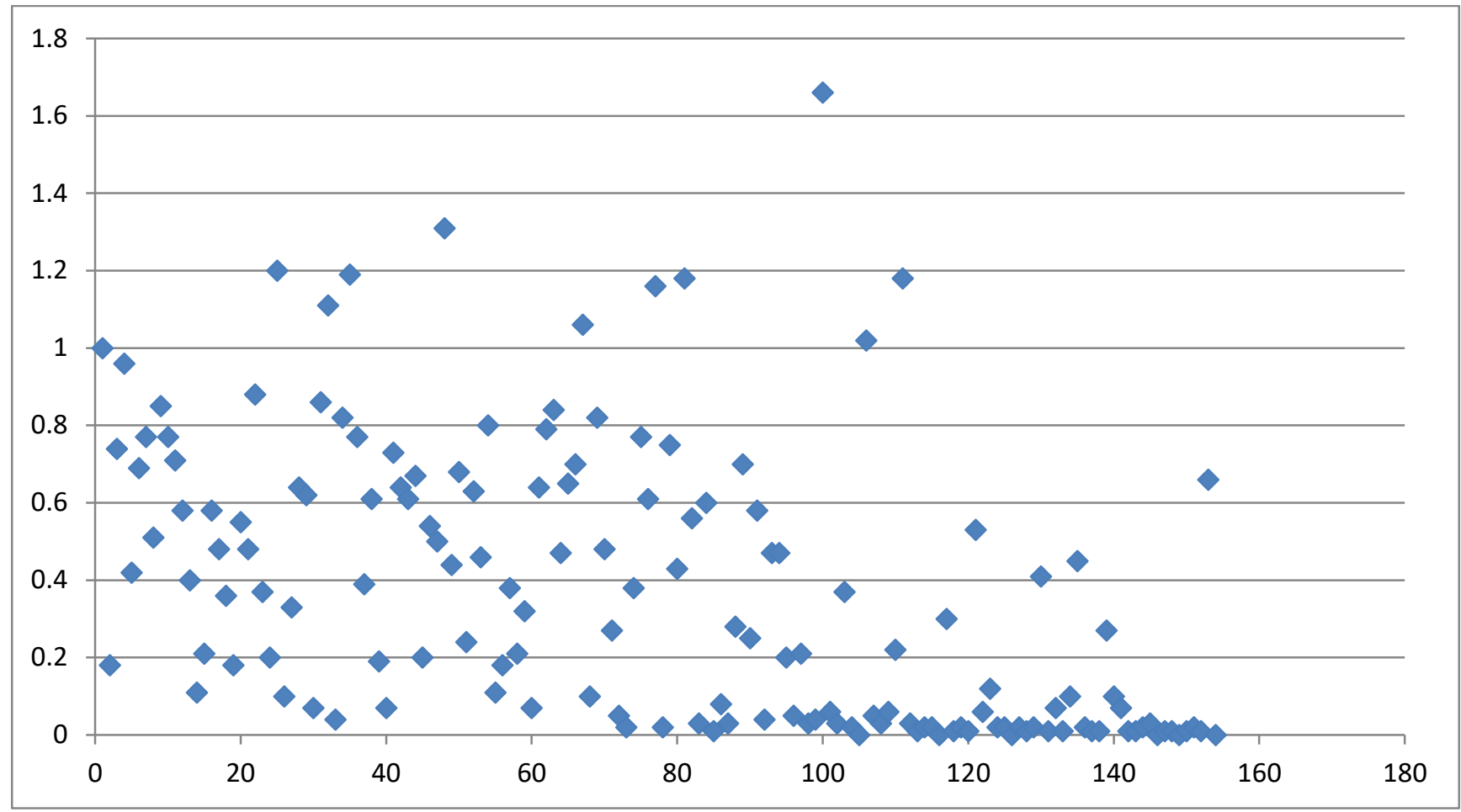

Figure 1: graph comparing infection case per country relative to USA

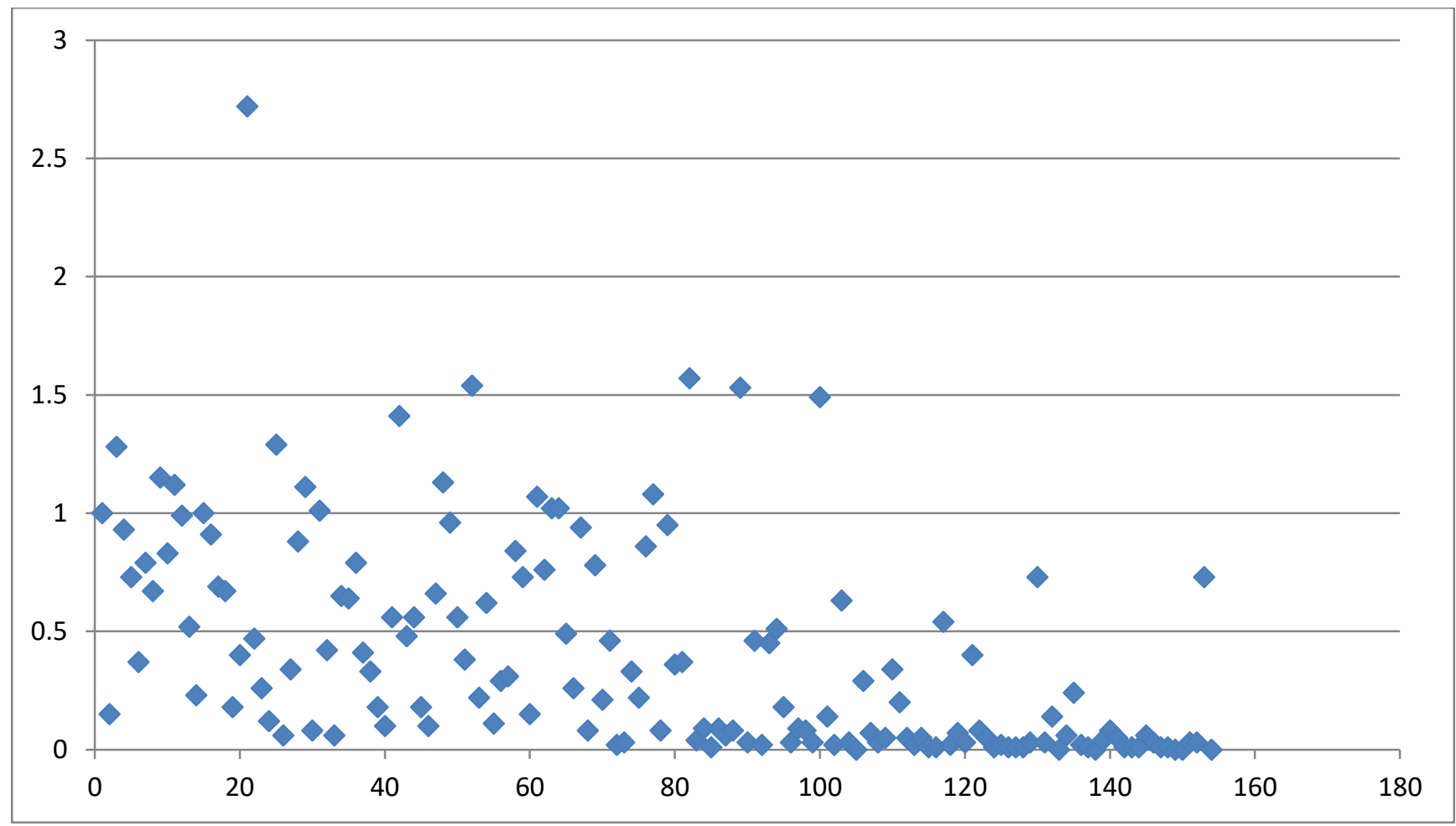

Figure 2: graph comparing infection case per country relative to USA

\section{DISCUSSION}

The COVID-19 pandemic, has affected hundreds of million persons, while it has resulted in the death of about five million human life worldwide. It also presents an unprecedented challenge to public health, food systems and the world of workforce [20]. The economic and social disruption caused by the pandemic is still unquantifiable. The highly infectious COVID-19 Delta variant has been indentified and reported in several countries, and it could affects hundreds and possibly thousands to millions of deaths if not properly contained[21]. Luckily enough, vaccines have recently been developed by different countries and companies to protect against the virus. The high cost of vaccination and low quantity produced and supplied relative to world population has 


\section{The Relative Global Consequences of Cumulative Distribution of Covid-19, Using the USA as Comparism Factor and Cumulative Covid -19 Data of 31 ${ }^{\text {st }}$ October 2021}

made it necessary to determine the distribution and availability of the vaccine across regions and countries based on relative urgency and emergency[22]. Hence, there is the need to understand the morphology, demographic and potential mechanisms of infectivity and adaptability in different countries.

Immunological studies have revealed a steady decline of antibody levels among vaccinated individuals[23,24]. Long-term followup of vaccine trial participants has revealed a growing risk of breakthrough infection[25]. And health-care records from countries such as Israel, the United Kingdom and elsewhere all show that COVID-19 vaccines could be losing their strength, at least when it comes to keeping a lid on transmissible disease. What remains unclear, however, is to what degree the immune system's safeguards that protect vaccinated people against severe disease, hospitalization and death might be fading as well. Neutralizing' antibodies that can intercept viruses before they infiltrate cells might not have much staying power. It has been observed that levels, of these molecules typically shoot up after vaccination, and then quickly taper off months later[26,27].

But cellular immune responses are longer lasting. Cellular immunity protect from the body disease. Memory B cells, which can rapidly deploy more antibodies in the event of re-exposure to the virus, tend to stick around, and so do T cells, which can attack already-infected cells[28,29]. Both provide an added measure of protection should SARS-CoV-2 sneak past the body's first line of defence. In one of the only long-term studies to consider these three planks of the immune system simultaneously - antibodies, B cells and T cells - researchers found that vaccination spurred durable cellular immunity[25]. Memory B cells continued to grow in numbers for at least six months period, and tends to get better at fighting the virus over time. T-cell counts remained relatively stable, dipping only slightly over the duration of the study period[30,31].

$B$ cells in these structures randomly mutate their genes to create entire new sets of antibodies. Those cells that produce the best antibody repertoires eventually win out through an evolutionary process that augments the immune system's ability to fight off Delta and other SARS-CoV-2 variants of concern. Real-world data from diagnostic-testing records and hospital databases suggest this view. In Israel, for example, elderly people who got their shots at the beginning of the year seemed to have almost double the risk of severe illness during a July outbreak compared with similar individuals who were immunized more recently[32]. As researchers recently reported that older individuals given a third dose of vaccine were less likely to be infected and much less likely to develop severe disease than individuals who have not received the boosters[33].

This study shows that Africa, known for poor health facilities, dreadfully unhealthy environment and indisputably still the world most underdeveloped region surprisingly has the least covid-19 infection and mortality case. Most African communities exist as a community and in dense clusters which is a strong contrast to most developed countries that are more solitary. Therefore, there is a higher chance that the virus may have circulated among high number of the Africa populace. Reasons for this positively unexpected figures has puzzled observers the world over. Previous works have attempted possible explanation for the globally available data. Environmental, Evolutionary and adaptive immune response to the virus may have caused low infectious and mortality case in Africa. It has also been observed that, African-American population is more affected by COVID-19 compared to other race in the USA $[34,35,36]$. Therefore, it is less likely to be genetic, but more likely related to the environment. Babraham Institute (2020) studied immune responses of African children compared to Dutch children. It was observed, that the immune systems of African children develop faster than those of Dutch children[37]. Exposure to bacteria, viral and fungi pathogens in childhood may have contributed to strengthen the immune system and protect children from developing allergies, asthma and other infectious diseases, on subsequence exposure to the same/similar allergen/pathogen or cross allergen/pathogen. This supports the 'hygiene hypothesis[38,39], which contends that such diseases are more common in the developed world where the prevalence of antibiotics and antibacterials reduce children's exposure to microbes[40,41]. Thus, early exposure to some diseases in Africa may have resulted in a more robust innate and/or adaptive immune response. As a result countries in Africa are both vulnerable and potentially more resilient to the coronavirus.

\section{CONCLUSION}

COVID-19, is not only a global pandemic and public health crisis; it has also severely affected the global economy and financial markets. At some point it caused the world to stand still, it struggles and watch how the virus unleashed it mayhem. African continent has been crippled by fear of possible consequences rather than the actual infection by the virus. Possible reasons why Africa is least affected in spite of popular expectations is yet to be fully understood. Also, Africa is a favourable home to several viral diseases such as chicken pox, Ebola, dengue fever, small pox, measles, and polio disease some of which the body system has found a way to developed and evolved to cope with. This may have caused direct or indirect cross immunity that later became beneficial on exposure to same, similar or different viral infection including corona virus disease.

Also, there may have been rapid transmission of the virus across the population within the shortest possible time. This means most Africans may have been exposed to the virus without showing noticeable symptoms and may have fully recovered, while 


\section{The Relative Global Consequences of Cumulative Distribution of Covid-19, Using the USA as Comparism Factor and Cumulative Covid -19 Data of 31 ${ }^{\text {st }}$ October 2021}

only few people have shown symptom to it. Therefore, there is need for COVID-19 antibody testing, which will reveal better picture of who has been exposed than the current antigen testing which only provides active disease information. This will immensely reduce the quantity and cost of vaccine that a give region may need.

The study also shows that, like every other continents, Africa needs vaccine, but in an emergency capacity when compared to western world its survival may not be desperately dependent on it, because most individuals in Africa countries may have been naturally and unconsciously immune.

\section{RECOMMENDATION}

More studies and surveys need to be conducted to understand the virus infectivity and it significances to Africa and maybe the rest of the world.

\section{CONFLICT OF INTEREST}

The authors declare that there are not any potential conflicts of interest

\section{ACKNOWLEDGEMENT}

The authors wish to appreciate and thank everyone who has contributed to the success of this study. Special appreciation to United Nations Geo scheme and WHO for access to raw data per country was gotten.

\section{AUTHORS' CONTRIBUTIONS}

Joseph OS and Joseph OT were involved in collection of data and development of model for analysis. Joseph OS, Joseph OT and Adeoye DA were responsible for analysis and writing of this manuscript.

\section{REFERENCE}

1) Nussbaumer-Streit B, Mayr V, Dobrescu Al, Chapman A, Persad E, Klerings I, et al. (April 2020). "Quarantine alone or in combination with other public health measures to control COVID-19: a rapid review". The Cochrane Database of Systematic Reviews. 4: Page 25-32

2) COVID-19 vaccines". World Health Organization (WHO). Retrieved 3 March 2021.

3) COVID-19 Dashboard by the Center for Systems Science and Engineering (CSSE) at Johns Hopkins University (JHU) . ArcGIS. Johns Hopkins University. Retrieved 3 November 2021.

4) Page J, Hinshaw D, McKay B (26 February 2021). In Hunt for Covid-19 Origin, Patient Zero Points to Second Wuhan Market - The man with the first confirmed infection of the new coronavirus told the WHO team that his parents had shopped there". The Wall Street Journal. Retrieved 27 February 2021.

5) Joseph O. S., Builders M., Joseph O. T., Sabastine A. Z. (2020). Assessing differential impacts of COVID-19 on African countries: A comparative study. International Journal of Research and Innovation in Applied Science. Vol. 5, Issue 5. Page 197-203

6) Joseph O S., Musa T L., Joseph O T. , Ibhafidon I. (2020). The Dynamics of Differential Impacts of COVID-19 on African Countries Compared to Other Parts of the World. International journal of multidisciplinary research and analysis. Volume 03 Issue 11. Page 185-198.

7) Joseph OS , Builders M , Joseph O T, Famojuro TI, Ogira JO, Moses FD, Musa TL. (2021). Effect of the Demographic of Covid-19 on Different Countries; Using the USA for Comparism. International journal of multidisciplinary research and analysis. Volume 04 Issue 02. Page 193-203.

8) Joseph O. S, Sabastine A. Z, Joseph O. T. (2021). Global Implication of Differential Impacts of Covid-19 on Different Countries Using the USA as A Comparism Factor. Journal of Nursing and Health Science. Volume 10, Issue 5. PP 36-44.

9) Zimmer C (26 February 2021). "The Secret Life of a Coronavirus - An oily, 100-nanometer-wide bubble of genes has killed more than two million people and reshaped the world. Scientists don't quite know what to make of it". Retrieved 28 February 2021.

10) Islam MA (April 2021). "Prevalence and characteristics of fever in adult and paediatric patients with coronavirus disease 2019 (COVID-19): A systematic review and meta-analysis of 17515 patients. PLOS ONE. 16 (4): Pg 224-234..

11) Joseph O. S, Sabastine A. Z, Joseph O. T. (2021). Clinical evaluation of the potential benefits of taking Moringa oleifera on blood triglyceride and cholesterol level in patient taking Tenofovir/Lamivudine/Efavirenz (TLE) combination. Journal of Pharmaceutical Science \& Research. Vol. 13(10), 623-629. 


\section{The Relative Global Consequences of Cumulative Distribution of Covid-19, Using the USA as Comparism Factor and}

Cumulative Covid -19 Data of 31 ${ }^{\text {st }}$ October 2021

12) Islam MA (November 2020). "Prevalence of Headache in Patients With Coronavirus Disease 2019 (COVID-19): A Systematic Review and Meta-Analysis of 14,275 Patients". Frontiers in Neurology. 11: 562634.

13) Saniasiaya J, Islam MA (April 2021). "Prevalence of Olfactory Dysfunction in Coronavirus Disease 2019 (COVID-19): A Meta-analysis of 27,492 Patients". The Laryngoscope. 131(4): 865-878.

14) Saniasiaya J, Islam MA (November 2020). "Prevalence and Characteristics of Taste Disorders in Cases of COVID-19: A Meta-analysis of 29,349 Patients". Otolaryngology-Head and Neck Surgery. 165 (1): 33-42.

15) Agyeman AA, Chin KL, Landersdorfer CB, Liew D, Ofori-Asenso R (August 2020). "Smell and Taste Dysfunction in Patients With COVID-19: A Systematic Review and Meta-analysis". Mayo Clin. Proc. 95 (8): 1621-1631.

16) Joseph O.S., Builders M., Emem E.U and Joseph O.T. (2019). effect of ethanol leaf extract of cassia angustifolia extract on liver of wister rats. Global Scientific Journal. Volume 8, Issue 9. Page 1112-11120.

17) Oran DP, Topol EJ (January 2021). "The Proportion of SARS-CoV-2 Infections That Are Asymptomatic : A Systematic Review". Annals of Internal Medicine. 174 (5): M20-6976.

18) Interim Clinical Guidance for Management of Patients with Confirmed Coronavirus Disease (COVID-19) . U.S. Centers for Disease Control and Prevention (CDC). 6 April 2020. Archived from the original on 2 March 2020. Retrieved 19 April 2020.

19) CDC (2020). Post-COVID Conditions. Centers for Disease Control and Prevention. Retrieved 12 July 2021.

20) Wu X, Nethery RC, Sabath MB, Braun D, Dominici F (November 2020). "Air pollution and COVID-19 mortality in the United States: Strengths and limitations of an ecological regression analysis". Science Advances. 6 (45): 342-355.

21) Joseph O.S, Builders M., Joseph O, T. , Zubairu S. A., Musa T. And Oyepata P. J (2019). Sub-Acute Toxicity Study of Ethanol Leaf Extract of Ocimum Canum on Liver of Wister Rats. International Journal of Research and Scientific Innovation. Volume VI (V). Pp. 364-369.

22) Oyebadejo S. A, Joseph O. S, Adesite S. O and Omorilewa A.O. (2019). Effect of Citrus Limon Juice and Tamoxifen on the Tumour growth mass Indices, Cell Proliferation, Cell Viability and Cytogenetic (Mitotic Index) of Sprague Dawley Rats Induced MCF-7 Breast Cancer Cells. Saudi Journal of Biomedical Research. (4). Pg. 216 - 225.

23) Modupe I. B., Oyepata S. J. and Akpobome R. V. (2019). Effect of Parkia biglobosa extract on open skin wound healing in dexamethasone - induced hyperglycaemia and histological assessment in rats. African Journal of Pharmacy and Pharmacology. Vol. 13(8), pp. 84-89.

24) Pansini R, Fornacca D (June 2021). Early Spread of COVID-19 in the Air-Polluted Regions of Eight Severely Affected Countries. Atmosphere. 12 (6): 795.

25) Comunian S, Dongo D, Milani C, Palestini P (June 2020). Air Pollution and Covid-19: The Role of Particulate Matter in the Spread and Increase of Covid-19's Morbidity and Mortality. International Journal of Environmental Research and Public Health. 17 (12): 4487.

26) Domingo JL, Marquès $M$, Rovira J (September 2020). "Influence of airborne transmission of SARS-CoV-2 on COVID-19 pandemic. A review". Environmental Research. 188: 66-73.

27) COVID-19: Who's at higher risk of serious symptoms? . Mayo Clinic.

28) Joseph O. S., Jude E.O and Joseph O. T (2018). Hepatoprotective activity of extract of Homalium Letestui stem against carbon tetrachloride-induced liver injury. Advance Herbal Medicine. Vol 4(4), Page 1-11.

29) Joseph O. S., Builders M., Joseph O. T., Zubairu S.A., Musa T. and Oyepata p.j. (2019). Sub-acute toxicity study of ethanol leaf extract of Ocimum canum on the kidney of wistar rats. African Journal of Pharmaceutical Research \& Development. Vol. 11 No.1. Page 1-7.

30) Tamara A, Tahapary DL (July 2020). "Obesity as a predictor for a poor prognosis of COVID-19: A systematic review". Diabetes \& Metabolic Syndrome. 14 (4): 655-659.

31) Petrakis D, Margină D, Tsarouhas K, Tekos F, Stan M, Nikitovic D, et al. (July 2020). Obesity - A risk factor for increased COVID-19, severity and lethality (Review) . Molecular Medicine Reports. 22 (1): 9-19.

32) Builders M., Joseph O. S., Timothy O. O., Philip B. (2020). Antimalarial Drugs and COVID -19. Sumerianz Journal of Medical and Healthcare. Vol. 3, No. 12, pp. 111-116.

33) Fan Y, Zhao K, Shi ZL, Zhou P. Bat Corona viruses in China. Viruses. 2019.11 (3): 210-223.

34) Jude E. O., Joseph O. S. and Emem E. U. (2016). Nephroprotective activity of Homalium letestui stem extract against paracetamol induced kidney injury. Journal of Experimental and Integrative Medicine. Volume 6 (1): 38-43.

35) Joseph O.S., Builders M., Emem E.U and Joseph O.T. (2019). effect of ethanol leaf extract of Cassia angustifolia extract on kidney of Wister Rats. Global Scientific Journal. Volume 8, Issue 9. Page 1023-1031. 
The Relative Global Consequences of Cumulative Distribution of Covid-19, Using the USA as Comparism Factor and Cumulative Covid -19 Data of 31 ${ }^{\text {st }}$ October 2021

36) Cherry, J, Demmler-Harrison GJ, Kaplan SL.; Steinbach WJ, Hotez PJ. Feign and Cherry's Textbook of Pediatric Infectious Diseases. Elsevier Health Sciences. 2017. p. 453-466.

37) Woo PC, Huang Y, Lau SK, Yuen KY. Corona virus genomics and bioinformatics analysis. Viruses. 2010. 2 (8): 1804-20.

38) Almeida JD, Berry DM, Cunningham CH, Hamre D, Hofstad MS, Mallucci L, McIntosh K, Tyrrell DA. Virology: Coronaviruses. Nature. 1968. 220 (5168): 265-278.

39) Jude E. O., Joseph O. S. and Emem E. U. (2016). Nephroprotective activity of Homalium letestui stem extract against paracetamol induced kidney injury. Journal of Experimental and Integrative Medicine. Volume 6 (1): 38-43.

40) https://www.cdc.gov/coronavirus/2019-ncov/transmission/variant.html

41) Chen N, Zhou M, Dong X, Qu J, Gong F, Han Y, et al. "Epidemiological and clinical characteristics of 99 cases of 2019 novel coronavirus pneumonia in Wuhan, China: a descriptive study". Lancet. 2020. 395 (10223): 507-513 